Proceedings of the Edinburgh Mathematical Society (2004) 47, 473-494 (C)

DOI:10.1017/S0013091503000701 Printed in the United Kingdom

\title{
ESTIMATES FOR MAHLER'S MEASURE OF A LINEAR FORM
}

\author{
FERNANDO RODRIGUEZ-VILLEGAS ${ }^{1}$, RICARDO TOLEDANO ${ }^{2}$ \\ AND JEFFREY D. VAALER ${ }^{1}$ \\ ${ }^{1}$ Department of Mathematics, University of Texas, Austin, TX 78712, USA \\ (villegas@math.utexas.edu; vaaler@math.utexas.edu) \\ ${ }^{2}$ Instituto de Matematica y Fisica, Universidad de Talca, \\ 2 Norte 685, Talca, Chile (toledano@inst-mat.utalca.cl)
}

(Received 19 August 2003)

\begin{abstract}
Let $L_{\boldsymbol{a}}(\boldsymbol{z})=a_{1} z_{1}+a_{2} z_{2}+\cdots+a_{N} z_{N}$ be a linear form in $N$ complex variables $z_{1}, z_{2}, \ldots, z_{N}$ with non-zero coefficients. We establish several estimates for the logarithmic Mahler measure of $L_{\boldsymbol{a}}$. In general, we show that the logarithmic Mahler measure of $L_{\boldsymbol{a}}(\boldsymbol{z})$ and the logarithm of the norm of $\boldsymbol{a}$ differ by a bounded amount that is independent of $N$. We prove a further estimate which is useful for making an approximate numerical evaluation of the logarithmic Mahler measure.
\end{abstract}

Keywords: Mahler measure; linear form; approximation

2000 Mathematics subject classification: Primary 11C08; 11Y35; 26D15

\section{Introduction}

Let $P(\boldsymbol{z})=P\left(z_{1}, z_{2}, \ldots, z_{N}\right)$ in $\mathbb{C}\left[z_{1}^{ \pm 1}, z_{2}^{ \pm 1}, \ldots, z_{N}^{ \pm 1}\right]$ be a Laurent polynomial in $N$ complex variables and let it not be identically zero. It can be shown by induction on $N$ (as in [7]) that the function $\boldsymbol{z} \rightarrow \log |P(\boldsymbol{z})|$ is integrable on the $N$-dimensional torus $\mathbb{T}^{N} \subseteq \mathbb{C}^{N}$ with respect to Haar measure. Alternatively, the function

$$
\boldsymbol{\theta} \rightarrow \log \left|P\left(e\left(\theta_{1}\right), e\left(\theta_{2}\right), \ldots, e\left(\theta_{N}\right)\right)\right|,
$$

where $e(\theta)=\mathrm{e}^{2 \pi \mathrm{i} \theta}$, is integrable on the group $(\mathbb{R} / \mathbb{Z})^{N}$ with respect to Haar measure. Then the value of the integral

$$
m(P)=\int_{(\mathbb{R} / \mathbb{Z})^{N}} \log \left|P\left(e\left(\theta_{1}\right), e\left(\theta_{2}\right), \ldots, e\left(\theta_{N}\right)\right)\right| \mathrm{d} \boldsymbol{\theta}
$$

defines the logarithmic Mahler measure of $P$. Here we will be interested in estimating the value of $m(P)$ in the special case where $P$ is a linear form. In a few cases there are explicit formulae for $m(P)$ which involve special values of certain $L$-series. For example, Smyth (see the appendix to [4]) has shown that

$$
\int_{(\mathbb{R} / \mathbb{Z})^{3}} \log \left|e\left(\theta_{1}\right)+e\left(\theta_{2}\right)+e\left(\theta_{3}\right)\right| \mathrm{d} \boldsymbol{\theta}=\frac{3 \sqrt{3}}{4 \pi} L(2, \chi),
$$


and

$$
\int_{(\mathbb{R} / \mathbb{Z})^{4}} \log \left|e\left(\theta_{1}\right)+e\left(\theta_{2}\right)+e\left(\theta_{3}\right)+e\left(\theta_{4}\right)\right| \mathrm{d} \boldsymbol{\theta}=\frac{7}{2 \pi^{2}} \zeta(3),
$$

where $\chi$ is the non-principal Dirichlet character to the modulus $3, L(s, \chi)$ is the corresponding Dirichlet $L$-function, and $\zeta(s)$ is the Riemann zeta-function. Further examples and conjectures have been given in $[\mathbf{1}],[\mathbf{5}],[\mathbf{3}],[\mathbf{6}]$ and $[\mathbf{9}]$. Explicit formulae similar to (1.1) and (1.2) are not known when $N$ is large. However, Smyth [10] and Myerson and Smyth $[\mathbf{8}]$ have established the estimate

$$
\int_{(\mathbb{R} / \mathbb{Z})^{N}} \log \left|e\left(\theta_{1}\right)+e\left(\theta_{2}\right)+\cdots+e\left(\theta_{N}\right)\right| \mathrm{d} \boldsymbol{\theta}=\frac{1}{2} \log N-\frac{1}{2} \gamma+O\left(\frac{\log N}{N}\right)
$$

as $N \rightarrow \infty$, where $\gamma$ is Euler's constant.

In the present paper we consider estimates for the logarithmic Mahler measure of a linear form:

$$
m\left(L_{\boldsymbol{a}}\right)=\int_{(\mathbb{R} / \mathbb{Z})^{N}} \log \left|a_{1} e\left(\theta_{1}\right)+a_{2} e\left(\theta_{2}\right)+\cdots+a_{N} e\left(\theta_{N}\right)\right| \mathrm{d} \boldsymbol{\theta},
$$

where $L_{\boldsymbol{a}}(\boldsymbol{z})=a_{1} z_{1}+a_{2} z_{2}+\cdots+a_{N} z_{N}$ and the coefficients $a_{1}, a_{2}, \ldots, a_{N}$ are not all zero. Of course, it is obvious that the integral (1.4) depends only on the numbers $\left|a_{1}\right|,\left|a_{2}\right|, \ldots,\left|a_{N}\right|$. To begin with we have the simple inequality [7, Exercise 3.3]

$$
\log |\boldsymbol{a}|_{\infty} \leqslant m\left(L_{\boldsymbol{a}}\right) \leqslant \log |\boldsymbol{a}|,
$$

where we use the vector space norms

$$
|\boldsymbol{a}|=\left(\left|a_{1}\right|^{2}+\left|a_{2}\right|^{2}+\cdots+\left|a_{N}\right|^{2}\right)^{1 / 2} \quad \text { and } \quad|\boldsymbol{a}|_{\infty}=\max \left\{\left|a_{1}\right|,\left|a_{2}\right|, \ldots,\left|a_{N}\right|\right\} .
$$

We are able to significantly improve the inequality on the left-hand side of (1.5).

Theorem 1.1. For all vectors $\boldsymbol{a} \neq \mathbf{0}$ in $\mathbb{C}^{N}$ we have

$$
\log |\boldsymbol{a}|-\frac{1}{2} \gamma-2 \leqslant m\left(L_{\boldsymbol{a}}\right) \leqslant \log |\boldsymbol{a}|,
$$

where $\gamma$ is Euler's constant.

It is clear that

$$
0=\inf \left\{\log |\boldsymbol{a}|-m\left(L_{\boldsymbol{a}}\right): \boldsymbol{a} \in \mathbb{C}^{N}, \boldsymbol{a} \neq \mathbf{0}\right\} .
$$

In view of the bound (1.6) it would be of interest to determine the non-decreasing sequence of numbers

$$
\delta_{N}=\sup \left\{\log |\boldsymbol{a}|-m\left(L_{\boldsymbol{a}}\right): \boldsymbol{a} \in \mathbb{C}^{N}, \boldsymbol{a} \neq \mathbf{0}\right\} \quad \text { and } \quad \lim _{N \rightarrow \infty} \delta_{N}=\Delta .
$$

It is trivial that $\delta_{1}=0$ and it is easy to show that $\delta_{2}=\frac{1}{2} \log 2$. As far as we know, the value of $\delta_{N}$ is not known for $3 \leqslant N$. Theorem 1.1 asserts that $\Delta \leqslant \frac{1}{2} \gamma+2$.

We obtain Theorem 1.1 from a more elaborate inequality. This shows that if the numbers $\left|a_{1}\right|,\left|a_{2}\right|, \ldots,\left|a_{N}\right|$ are not dominated by a few large terms, then $m\left(L_{\boldsymbol{a}}\right)$ is approximately equal to $\log |\boldsymbol{a}|-\frac{1}{2} \gamma$. 
Theorem 1.2. Let $3 \leqslant N$ and let $\boldsymbol{a}$ be a non-zero vector in $\mathbb{C}^{N}$. Then we have

$$
\begin{aligned}
-2 \mid\{n: & \left.\leqslant n \leqslant N,|\boldsymbol{a}|_{\infty} \leqslant \pi\left|a_{n}\right|\right\}\left.\right|^{-1} \\
& \leqslant m\left(L_{\boldsymbol{a}}\right)-\log |\boldsymbol{a}|+\frac{1}{2} \gamma \\
& \leqslant \frac{\left|a_{1}\right|^{4}+\left|a_{2}\right|^{4}+\cdots+\left|a_{N}\right|^{4}}{4|\boldsymbol{a}|^{4}}+4\left|\left\{n: 1 \leqslant n \leqslant N,|\boldsymbol{a}|_{\infty} \leqslant \pi\left|a_{n}\right|\right\}\right|^{-1} .
\end{aligned}
$$

In the special case $a_{1}=a_{2}=\cdots=a_{N}=1$ the estimate (1.8) clearly implies that

$$
\int_{(\mathbb{R} / \mathbb{Z})^{N}} \log \left|e\left(\theta_{1}\right)+e\left(\theta_{2}\right)+\cdots+e\left(\theta_{N}\right)\right| \mathrm{d} \boldsymbol{\theta}=\frac{1}{2} \log N-\frac{1}{2} \gamma+O\left(N^{-1}\right),
$$

which slightly sharpens (1.3). We conjecture that the error term on the right-hand side of (1.9) can be replaced by an asymptotic expansion that we determine in (4.21). Theorem 1.2 allows us to obtain essentially the same estimate for more general classes of linear forms. As an example, let $p(z)$ be a non-zero polynomial in $\mathbb{C}[z]$. Then for all sufficiently large integers $N$ we find that

$$
\begin{aligned}
\int_{(\mathbb{R} / \mathbb{Z})^{N}} \log \left|p(1) e\left(\theta_{1}\right)+p(2) e\left(\theta_{2}\right)+\cdots+p(N) e\left(\theta_{N}\right)\right| \mathrm{d} \boldsymbol{\theta} \\
=\frac{1}{2} \log \left\{|p(1)|^{2}+|p(2)|^{2}+\cdots+|p(N)|^{2}\right\}-\frac{1}{2} \gamma+O_{p}\left(N^{-1}\right),
\end{aligned}
$$

where the constant implied by $O_{p}$ depends on $p$.

Now suppose that $b_{0}, b_{1}, \ldots, b_{N}$ are complex numbers with $b_{0} \neq 0$. From Jensen's formula we find that

$$
\begin{aligned}
& \int_{(\mathbb{R} / \mathbb{Z})^{N+1}} \log \left|b_{0} e\left(\theta_{0}\right)+b_{1} e\left(\theta_{1}\right)+\cdots+b_{N} e\left(\theta_{N}\right)\right| \mathrm{d} \boldsymbol{\theta} \\
& \quad=\log \left|b_{0}\right|+\int_{(\mathbb{R} / \mathbb{Z})^{N}} \log ^{+}\left|b_{0}^{-1} b_{1} e\left(\theta_{1}\right)+b_{0}^{-1} b_{2} e\left(\theta_{2}\right)+\cdots+b_{0}^{-1} b_{N} e\left(\theta_{N}\right)\right| \mathrm{d} \boldsymbol{\theta},
\end{aligned}
$$

where $\log ^{+} x=\max \{0, \log x\}$ for positive real $x$. Thus, for the purpose of estimating the Mahler measure of a linear form, it suffices to consider integrals of the form

$$
\int_{(\mathbb{R} / \mathbb{Z})^{N}} \log ^{+}\left|a_{1} e\left(\theta_{1}\right)+a_{2} e\left(\theta_{2}\right)+\cdots+a_{N} e\left(\theta_{N}\right)\right| \mathrm{d} \boldsymbol{\theta} .
$$

We now state a result which can be used to obtain good numerical approximations to (1.12). In doing so we make use of the vector space norm

$$
|\boldsymbol{a}|_{1}=\left|a_{1}\right|+\left|a_{2}\right|+\cdots+\left|a_{N}\right| .
$$

Let $0<\alpha_{1}<\alpha_{2}<\cdots$ be the consecutive positive zeros of the Bessel function $J_{0}(z)$. If $\boldsymbol{a}$ is a non-zero vector in $\mathbb{C}^{N}$ and $l$ is a positive integer, we define

$$
\psi(\boldsymbol{a}, l)=2 \alpha_{l}^{-1} J_{1}\left(\alpha_{l}\right)^{-2} \sum_{m=1}^{N} q_{m} J_{1}\left(\alpha_{l} q_{m}\right) \prod_{\substack{n=1 \\ n \neq m}}^{N} J_{0}\left(\alpha_{l} q_{n}\right),
$$


where we have written

$$
q_{m}=\left|a_{m}\right||\boldsymbol{a}|_{1}^{-1} \quad \text { for } m=1,2, \ldots, N .
$$

Theorem 1.3. Let $3 \leqslant N$ and let $\boldsymbol{a}$ be a vector in $\mathbb{C}^{N}$ with $1 \leqslant|\boldsymbol{a}|_{1}$. Then we have

$$
\begin{aligned}
\int_{(\mathbb{R} / \mathbb{Z})^{N}} \log ^{+} \mid a_{1} e\left(\theta_{1}\right)+a_{2} e\left(\theta_{2}\right)+ & \cdots+a_{N} e\left(\theta_{N}\right) \mid \mathrm{d} \boldsymbol{\theta} \\
& =\sum_{l=1}^{\infty} \psi(\boldsymbol{a}, l) \int_{1}^{|\boldsymbol{a}|_{1}} J_{0}\left(\alpha_{l}|\boldsymbol{a}|_{1}^{-1} u\right) u^{-1} \mathrm{~d} u .
\end{aligned}
$$

To obtain a good numerical approximation to $m\left(L_{a}\right)$ we require an estimate for the tail of the infinite series on the right-hand side of (1.14).

Corollary 1.4. Let $3 \leqslant N$ and let

$$
Q=\left(q_{1} q_{2} \cdots q_{N}\right)^{1 / N}
$$

denote the geometric mean of the numbers $q_{n}=\left|a_{n}\right||\boldsymbol{a}|_{1}^{-1}$. For $5 \leqslant L$ the tail of the series on the right-hand side of (1.14) satisfies the inequality

$$
\sum_{l=L+1}^{\infty}\left|\psi(\boldsymbol{a}, l) \int_{1}^{|\boldsymbol{a}|_{1}} J_{0}\left(\alpha_{l}|\boldsymbol{a}|_{1}^{-1} u\right) u^{-1} \mathrm{~d} u\right| \leqslant|\boldsymbol{a}|_{1}^{3 / 2}\left(\frac{26}{N}\right)\left(\frac{2}{\pi^{2} Q}\right)^{N / 2}\left(\frac{1}{L}\right)^{(N+1) / 2} .
$$

As an application of Corollary 1.4, let $K_{N}(\boldsymbol{z})$ denote the linear form

$$
K_{N}(\boldsymbol{z})=z_{1}+z_{2}+\cdots+z_{N}
$$

Then for $5 \leqslant N \leqslant 20$ we find the following numerical approximations to the logarithmic Mahler measure $m\left(K_{N}\right)$ :

$$
\begin{aligned}
& m\left(K_{5}\right)=0.544412561752185 \ldots, \quad m\left(K_{13}\right)=1.003583530489340 \ldots, \\
& m\left(K_{6}\right)=0.627317074836909 \ldots, \quad m\left(K_{14}\right)=1.039935308341516 \ldots, \\
& m\left(K_{7}\right)=0.702926292476967 \ldots, \quad m\left(K_{15}\right)=1.073826217256882 \ldots, \\
& m\left(K_{8}\right)=0.766831088069615 \ldots, \quad m\left(K_{16}\right)=1.105565343200777 \ldots, \\
& m\left(K_{9}\right)=0.824156239532393 \ldots, \quad m\left(K_{17}\right)=1.135410703767443 \ldots, \\
& m\left(K_{10}\right)=0.875328658114493 \ldots, \quad m\left(K_{18}\right)=1.163575074215941 \ldots, \\
& m\left(K_{11}\right)=0.921850886732666 \ldots, \quad m\left(K_{19}\right)=1.190237864644479 \ldots, \\
& m\left(K_{12}\right)=0.964375789319576 \ldots, \quad m\left(K_{20}\right)=1.215550991648849 \ldots,
\end{aligned}
$$

We note that Equation (4.7) of Theorem 4.1 also contains an identity for the Mahler measure of a linear form. At the end of $\S 4$ we state a conjecture which, together with (4.7), would provide a simpler method for estimating the numbers $m\left(K_{N}\right)$. 


\section{Preliminary lemmas}

For each integer $m=0,1,2, \ldots$ let

$$
L_{m}(x)=\sum_{l=0}^{m}\left(\begin{array}{c}
m \\
l
\end{array}\right) \frac{(-x)^{l}}{l !}
$$

denote the corresponding Laguerre polynomial, and write

$$
\mathcal{L}_{m}(x)=L_{m}(x) \mathrm{e}^{-x / 2}
$$

for the associated Laguerre function. Let $s=\sigma+$ it denote a complex variable.

Lemma 2.1. In the half-plane $\{s \in \mathbb{C}:-1<\operatorname{Re}(s)\}$ we have

$$
\int_{0}^{\infty} x^{s} L_{m}(x) \mathrm{e}^{-x} \mathrm{~d} x=(-1)^{m} \Gamma(s+1)\left(\begin{array}{c}
s \\
m
\end{array}\right) .
$$

Proof. From the Rodrigues formula we get

$$
\int_{0}^{\infty} x^{s} L_{m}(x) \mathrm{e}^{-x} \mathrm{~d} x=\int_{0}^{\infty} x^{s}\left\{D^{(m)} \mathrm{e}^{-x} x^{m}\right\} \mathrm{d} x,
$$

where

$$
D^{(m)}=\frac{1}{m !}\left(\frac{\mathrm{d}}{\mathrm{d} x}\right)^{m}
$$

Then the identity (2.2) follows after integrating by parts $m$ times.

Corollary 2.2. For each integer $m=0,1,2, \ldots$ we have

$$
\int_{0}^{\infty}(\log x) L_{m}(x) \mathrm{e}^{-x} \mathrm{~d} x= \begin{cases}-\gamma & \text { if } m=0, \\ -m^{-1} & \text { if } m=1,2, \ldots,\end{cases}
$$

where $\gamma$ is Euler's constant.

Proof. This follows by differentiating both sides of (2.2), setting $s=0$, and using $\Gamma^{\prime}(1)=-\gamma$.

The function $x \rightarrow(\log x) \mathrm{e}^{-x / 2}$ obviously belongs to $L^{2}\{[0, \infty)\}$. As the functions $\left\{\mathcal{L}_{0}(x), \mathcal{L}_{1}(x), \ldots\right\}$ form a complete orthonormal system in $L^{2}\{[0, \infty)\}$, we find that

$$
(\log x) \mathrm{e}^{-x / 2}=\lim _{M \rightarrow \infty} \sum_{m=0}^{M} a_{m} \mathcal{L}_{m}(x)
$$

in $L^{2}$-norm on $[0, \infty)$, where the coefficients $a_{m}$ are determined by

$$
a_{m}=\int_{0}^{\infty}(\log x) \mathrm{e}^{-x / 2} \mathcal{L}(x) \mathrm{d} x= \begin{cases}-\gamma & \text { if } m=0 \\ -m^{-1} & \text { if } m=1,2, \ldots\end{cases}
$$


Thus we have

$$
(\log x) \mathrm{e}^{-x / 2}=-\gamma \mathrm{e}^{-x / 2}-\lim _{M \rightarrow \infty} \mathrm{e}^{-x / 2} \sum_{m=1}^{M} m^{-1} L_{m}(x)
$$

in $L^{2}$-norm on $[0, \infty)$.

Lemma 2.3. For all $\boldsymbol{x}$ in $\mathbb{R}^{N}$ such that $|\boldsymbol{x}|_{\infty} \leqslant 1$, we have

$$
0 \leqslant \exp \left\{-|\boldsymbol{x}|^{2}\right\}-\prod_{n=1}^{N} J_{0}\left(2 x_{n}\right) \leqslant \frac{1}{2} \exp \left\{-|\boldsymbol{x}|^{2}\right\}\left\{x_{1}^{4}+x_{2}^{4}+\cdots+x_{N}^{4}\right\} .
$$

Proof. Let $0<\alpha_{1}<\alpha_{2}<\cdots$ denote the positive real zeros of the Bessel function $J_{0}(z)$, so that

$$
J_{0}(z)=\lim _{L \rightarrow \infty} \prod_{l=1}^{L}\left(1-\frac{z^{2}}{\alpha_{l}^{2}}\right)
$$

uniformly on compact subsets of $\mathbb{C}$. Then define

$$
\beta_{m}=\sum_{l=1}^{\infty}\left(2 / \alpha_{l}\right)^{2 m} \text { for } m=1,2, \ldots
$$

The numbers $\beta_{m}$ are rational,

$$
\beta_{1}=1, \quad \beta_{2}=\frac{1}{2}, \quad \beta_{3}=\frac{1}{3}, \quad \beta_{4}=\frac{11}{48}, \quad \beta_{5}=\frac{19}{120}, \quad \beta_{6}=\frac{473}{4320},
$$

and were calculated by Euler [13, pp. 500, 501]. In the open disc $\left\{z:|z|<\frac{1}{2} \alpha_{1}\right\}$ we have

$$
\begin{aligned}
\log J_{0}(2 z) & =\sum_{l=1}^{\infty} \log \left(1-\frac{4 z^{2}}{\alpha_{l}^{2}}\right) \\
& =-\sum_{l=1}^{\infty} \sum_{m=1}^{\infty} m^{-1}\left(\frac{2 z}{\alpha_{l}}\right)^{2 m} \\
& =-\sum_{m=1}^{\infty} m^{-1} \beta_{m} z^{2 m}
\end{aligned}
$$

We note for later use that

$$
\sum_{m=2}^{\infty} m^{-1} \beta_{m}=\log J_{0}(2)-1 \leqslant \frac{1}{2}
$$

Let $\boldsymbol{x}$ be a vector in $\mathbb{R}^{N}$, and assume that $|\boldsymbol{x}|_{\infty} \leqslant 1$. Using (2.7) we obtain the identity

$$
\begin{aligned}
\sum_{n=1}^{N}\left(x_{n}^{2}+\log J_{0}\left(2 x_{n}\right)\right) & =\sum_{n=1}^{N}\left\{x_{n}^{2}-\sum_{m=1}^{\infty} m^{-1} \beta_{m} x_{n}^{2 m}\right\} \\
& =-\sum_{m=2}^{\infty} m^{-1} \beta_{m} S_{m}(\boldsymbol{x})
\end{aligned}
$$


where we have written

$$
S_{m}(\boldsymbol{x})=x_{1}^{2 m}+x_{2}^{2 m}+\cdots+x_{N}^{2 m}
$$

Using (2.9) we get

$$
\begin{aligned}
\exp \left\{-|\boldsymbol{x}|^{2}\right\}-\prod_{n=1}^{N} J_{0}\left(2 x_{n}\right) & =\exp \left\{-|\boldsymbol{x}|^{2}\right\}\left(1-\exp \left\{\sum_{n=1}^{N}\left(x_{n}^{2}+\log J_{0}\left(2 x_{n}\right)\right)\right\}\right) \\
& =\exp \left\{-|\boldsymbol{x}|^{2}\right\}\left(1-\exp \left\{-\sum_{m=2}^{\infty} m^{-1} \beta_{m} S_{m}(\boldsymbol{x})\right\}\right)
\end{aligned}
$$

This verifies the lower bound on the left-hand side of (2.5). To establish the upper bound we use (2.8). We find that

$$
\begin{aligned}
\sum_{m=2}^{\infty} m^{-1} \beta_{m} S_{m}(\boldsymbol{x}) & =\sum_{n=1}^{N}\left\{x_{n}^{4} \sum_{m=2}^{\infty} m^{-1} \beta_{m} x_{n}^{2 m-4}\right\} \\
& \leqslant \sum_{n=1}^{N}\left\{x_{n}^{4} \sum_{m=2}^{\infty} m^{-1} \beta_{m}\right\} \\
& \leqslant \frac{1}{2} S_{2}(\boldsymbol{x}),
\end{aligned}
$$

and then

$$
1-\exp \left\{-\frac{1}{2} S_{2}(\boldsymbol{x})\right\} \leqslant \frac{1}{2} S_{2}(\boldsymbol{x})
$$

The inequality on the right-hand side of (2.5) follows from (2.10), (2.11) and (2.12).

\section{Sums of random vectors}

Let $\boldsymbol{X}_{1}, \boldsymbol{X}_{2}, \ldots, \boldsymbol{X}_{N}$ be independent random vectors uniformly distributed on the unit circle in $\mathbb{R}^{2}$. Then let $\boldsymbol{Y}$ be the random vector

$$
\boldsymbol{Y}=r_{1} \boldsymbol{X}_{1}+r_{2} \boldsymbol{X}_{2}+\cdots+r_{N} \boldsymbol{X}_{N}
$$

where $r_{1}, r_{2}, \ldots, r_{N}$ are positive real numbers. The random vector $\boldsymbol{Y}$ induces a probability measure $\mu_{\boldsymbol{Y}}$ on the Borel subsets of $\mathbb{R}^{2}$. For $2 \leqslant N$ this measure is absolutely continuous with respect to Haar measure on the additive group of $\mathbb{R}^{2}$. We assume that $3 \leqslant N$ and write $f_{\boldsymbol{r}}(\boldsymbol{x})$ for the associated probability density function. Thus we have

$$
\operatorname{Prob}\{\boldsymbol{Y} \in B\}=\mu_{\boldsymbol{Y}}(B)=\int_{B} f_{\boldsymbol{r}}(\boldsymbol{x}) \mathrm{d} \boldsymbol{x}
$$

for all Borel subsets $B \subseteq \mathbb{R}^{2}$. It is clear that the function $\boldsymbol{x} \rightarrow f_{\boldsymbol{r}}(\boldsymbol{x})$ is radial and it will be convenient to define $\tilde{f}_{\boldsymbol{r}}:[0, \infty) \rightarrow[0, \infty)$ by $\tilde{f}_{\boldsymbol{r}}(|\boldsymbol{x}|)=f_{\boldsymbol{r}}(\boldsymbol{x})$. We note that $f_{\boldsymbol{r}}$ is supported on the closed disc of radius $|\boldsymbol{r}|_{1}$ and $\tilde{f}_{\boldsymbol{r}}$ is supported on the interval $\left[0,|\boldsymbol{r}|_{1}\right]$. We also define the function $F_{\boldsymbol{r}}: \mathbb{R}^{2} \rightarrow[0,1]$ by

$$
F_{\boldsymbol{r}}(\boldsymbol{y})=\operatorname{Prob}\{|\boldsymbol{Y}| \leqslant|\boldsymbol{y}|\}=\int_{|\boldsymbol{x}| \leqslant|\boldsymbol{y}|} f_{\boldsymbol{r}}(\boldsymbol{x}) \mathrm{d} \boldsymbol{x}=2 \pi \int_{0}^{|\boldsymbol{y}|} u \tilde{f}_{\boldsymbol{r}}(u) \mathrm{d} u,
$$


and the probability distribution function $\tilde{F}_{\boldsymbol{r}}:[0, \infty) \rightarrow[0,1]$ by

$$
\tilde{F}_{\boldsymbol{r}}(|\boldsymbol{y}|)=F_{\boldsymbol{r}}(\boldsymbol{y}) \quad \text { or } \quad \tilde{F}_{\boldsymbol{r}}(u)=2 \pi \int_{0}^{u} v \tilde{f}_{\boldsymbol{r}}(v) \mathrm{d} v .
$$

Let $\mu_{\boldsymbol{X}}$ be the probability measure induced by a random vector $\boldsymbol{X}$ uniformly distributed on the unit circle. Then the Fourier transform of $\mu_{\boldsymbol{X}}$ is

$$
\begin{aligned}
\hat{\mu}_{\boldsymbol{X}}(\boldsymbol{t}) & =\int_{\mathbb{R}^{2}} e(-\boldsymbol{t} \boldsymbol{x}) \mathrm{d} \mu_{\boldsymbol{X}}(\boldsymbol{x}) \\
& =\int_{\mathbb{R} / \mathbb{Z}} e\left(-t_{1} \cos 2 \pi \theta-t_{2} \sin 2 \pi \theta\right) \mathrm{d} \theta=J_{0}(2 \pi|\boldsymbol{t}|) .
\end{aligned}
$$

It follows that the Fourier transform of the measure $\mu_{\boldsymbol{Y}}$ and the associated density function $f_{\boldsymbol{r}}(\boldsymbol{x})$ is given by

$$
\hat{\mu}_{\boldsymbol{Y}}(\boldsymbol{t})=\hat{f}_{\boldsymbol{r}}(\boldsymbol{t})=\int_{\mathbb{R}^{2}} f_{\boldsymbol{r}}(\boldsymbol{x}) e(-\boldsymbol{t} \boldsymbol{x}) \mathrm{d} \boldsymbol{x}=\prod_{n=1}^{N} J_{0}\left(2 \pi r_{n}|\boldsymbol{t}|\right) .
$$

Lemma 3.1. If $3 \leqslant N$, then the density function $f_{\boldsymbol{r}}(\boldsymbol{x})$ belongs to $L^{2}\left(\mathbb{R}^{2}\right)$.

Proof. We use the well-known inequality [12, Theorem 7.31.2]

$$
\left|J_{0}(2 x)\right| \leqslant(\max \{1,|\pi x|\})^{-1 / 2},
$$

which holds for all real $x$. Then from (3.4) and Plancherel's identity we get

$$
\begin{aligned}
\int_{\mathbb{R}^{2}}\left|f_{\boldsymbol{r}}(\boldsymbol{y})\right|^{2} \mathrm{~d} \boldsymbol{y} & =\int_{\mathbb{R}^{2}} \prod_{n=1}^{N}\left|J_{0}\left(2 \pi r_{n}|\boldsymbol{t}|\right)\right|^{2} \mathrm{~d} \boldsymbol{t} \\
& =2 \pi \int_{0}^{\infty} u \prod_{n=1}^{N}\left|J_{0}\left(2 \pi r_{n} u\right)\right|^{2} \mathrm{~d} u \\
& \leqslant 2 \pi \int_{0}^{\infty} u \prod_{n=1}^{N}\left(\max \left\{1,\left|\pi r_{n} u\right|\right\}\right)^{-1} \mathrm{~d} u \\
& \ll \int_{0}^{\infty} u(\max \{1,|u|\})^{-N} \mathrm{~d} u \\
& <\infty .
\end{aligned}
$$

As $f_{\boldsymbol{r}}(\boldsymbol{x})$ is a radial function on $\mathbb{R}^{2}$, its transform $\hat{f}_{\boldsymbol{r}}(\boldsymbol{t})$ is also radial and is given by [11, Chapter IV, Theorem 3.3]

$$
\hat{f}_{\boldsymbol{r}}(\boldsymbol{t})=\int_{\mathbb{R}^{2}} f_{\boldsymbol{r}}(\boldsymbol{x}) J_{0}(2 \pi|\boldsymbol{t}||\boldsymbol{x}|) \mathrm{d} \boldsymbol{x}=2 \pi \int_{0}^{\infty} u \tilde{f}_{\boldsymbol{r}}(u) J_{0}(2 \pi|\boldsymbol{t}| u) \mathrm{d} u .
$$


For our purposes it is useful to observe that for all complex $z$ the function

$$
u \rightarrow u \tilde{f}_{\boldsymbol{r}}(u) J_{0}(2 \pi z u)
$$

is bounded and compactly supported on $[0, \infty)$. Hence the integral

$$
z \rightarrow \int_{0}^{\infty} u \tilde{f}_{\boldsymbol{r}}(u) J_{0}(2 \pi u z) \mathrm{d} u
$$

is defined for all complex $z$. Then it follows, using Morera's Theorem, that (3.7) defines an entire function of $z$.

Lemma 3.2. The identity

$$
\prod_{n=1}^{N} J_{0}\left(2 \pi r_{n} z\right)=2 \pi \int_{0}^{\infty} u \tilde{f}_{\boldsymbol{r}}(u) J_{0}(2 \pi z u) \mathrm{d} u
$$

holds for all complex $z$.

Proof. Both sides of (3.8) define entire functions. Using (3.4) and (3.6) we see that these functions are equal on the positive real axis. Hence they are equal on $\mathbb{C}$.

For all complex $z$ the function

$$
u \rightarrow u\left(1-\tilde{F}_{\boldsymbol{r}}(u)\right) J_{0}(2 \pi u z)
$$

is also compactly supported on $[0, \infty)$. In a similar manner we find that

$$
z \rightarrow \int_{0}^{\infty} u\left(1-\tilde{F}_{\boldsymbol{r}}(u)\right) J_{0}(2 \pi u z) \mathrm{d} u
$$

defines an entire function of $z$.

Lemma 3.3. The identity

$$
\sum_{m=1}^{N} r_{m} J_{1}\left(2 \pi r_{m} z\right) \prod_{\substack{n=1 \\ n \neq m}}^{N} J_{0}\left(2 \pi r_{n} z\right)=2 \pi z \int_{0}^{\infty} u\left(1-\tilde{F}_{\boldsymbol{r}}(u)\right) J_{0}(2 \pi z u) \mathrm{d} u
$$

holds for all complex $z$.

Proof. By differentiating both sides of (3.8) we obtain the identity

$$
\sum_{m=1}^{N} r_{m} J_{1}\left(2 \pi r_{m} z\right) \prod_{\substack{n \neq 1 \\ n \neq m}}^{N} J_{0}\left(2 \pi r_{n} z\right)=2 \pi \int_{0}^{\infty} u^{2} \tilde{f}_{\boldsymbol{r}}(u) J_{1}(2 \pi z u) \mathrm{d} u .
$$

Next we use the identity on the right-hand side of (3.3) and find that

$$
\frac{\mathrm{d}}{\mathrm{d} u} u\left(1-\tilde{F}_{\boldsymbol{r}}(u)\right) J_{1}(2 \pi z u)=2 \pi z u\left(1-\tilde{F}_{\boldsymbol{r}}(u)\right) J_{0}(2 \pi z u)-2 \pi u^{2} \tilde{f}_{\boldsymbol{r}}(u) J_{1}(2 \pi z u)
$$

at almost all points $u$ in $[0, \infty)$. Hence we have

$$
2 \pi z \int_{0}^{\infty} u\left(1-\tilde{F}_{\boldsymbol{r}}(u)\right) J_{0}(2 \pi z u) \mathrm{d} u=2 \pi \int_{0}^{\infty} u^{2} \tilde{f}_{\boldsymbol{r}}(u) J_{1}(2 \pi z u) \mathrm{d} u .
$$

The result now follows by combining (3.10) and (3.11). 


\section{Identities for Mahler's measure}

Let $\boldsymbol{\theta}$ denote a point in the group $(\mathbb{R} / \mathbb{Z})^{N}$ with Haar measure normalized so that $(\mathbb{R} / \mathbb{Z})^{N}$ has measure 1 . If we identify $\mathbb{C}$ and $\mathbb{R}^{2}$, the coordinate functions $\boldsymbol{\theta} \rightarrow e\left(\theta_{n}\right)$ provide an example of $N$ independent random vectors uniformly distributed on the unit circle. Then it follows in a standard manner from (3.1) that

$$
\begin{aligned}
\int_{(\mathbb{R} / \mathbb{Z})^{N}} T\left(\left|r_{1} e\left(\theta_{1}\right)+r_{2} e\left(\theta_{2}\right)+\cdots+r_{N} e\left(\theta_{N}\right)\right|\right) \mathrm{d} \boldsymbol{\theta} & =\int_{\mathbb{R}^{2}} f_{\boldsymbol{r}}(\boldsymbol{x}) T(|\boldsymbol{x}|) \mathrm{d} \boldsymbol{x} \\
& =2 \pi \int_{0}^{\infty} u \tilde{f}_{\boldsymbol{r}}(u) T(u) \mathrm{d} u
\end{aligned}
$$

for all continuous functions $T:[0, \infty) \rightarrow \mathbb{C}$. Now let $s=\sigma+$ it be a complex variable. As an application of (4.1), in the half-plane $\{s \in \mathbb{C}: 0<\operatorname{Re}(s)\}$ we define

$$
\begin{aligned}
\Lambda_{\boldsymbol{r}}(s) & =\int_{(\mathbb{R} / \mathbb{Z})^{N}}\left|r_{1} e\left(x_{1}\right)+r_{2} e\left(x_{2}\right)+\cdots+r_{N} e\left(x_{N}\right)\right|^{2 s} \mathrm{~d} \boldsymbol{x} \\
& =\int_{\mathbb{R}^{2}}|\boldsymbol{y}|^{2 s} f_{\boldsymbol{r}}(\boldsymbol{y}) \mathrm{d} \boldsymbol{y} \\
& =2 \pi \int_{0}^{\infty} u^{2 s+1} \tilde{f}_{\boldsymbol{r}}(u) \mathrm{d} u .
\end{aligned}
$$

It will be convenient to set

$$
\varphi_{\boldsymbol{r}}(u)=\pi|\boldsymbol{r}|^{2} \tilde{f}_{\boldsymbol{r}}(|\boldsymbol{r}| \sqrt{u}) \text { for } 0 \leqslant u .
$$

Then (4.2) can be written as

$$
\Lambda_{\boldsymbol{r}}(s)=|\boldsymbol{r}|^{2 s} \int_{0}^{\infty} v^{s} \varphi_{\boldsymbol{r}}(v) \mathrm{d} v .
$$

If $3 \leqslant N$, then from Lemma 3.1 we have

$$
\begin{aligned}
\pi^{-1}|\boldsymbol{r}|^{-2} \int_{0}^{\infty}\left|\varphi_{\boldsymbol{r}}(u)\right|^{2} \mathrm{~d} u & =2 \pi \int_{0}^{\infty} v\left|\tilde{f}_{\boldsymbol{r}}(v)\right|^{2} \mathrm{~d} v \\
& =\int_{\mathbb{R}^{2}}\left|f_{\boldsymbol{r}}(\boldsymbol{x})\right|^{2} \mathrm{~d} \boldsymbol{x}<\infty
\end{aligned}
$$

As $\varphi_{\boldsymbol{r}}(u)$ is compactly supported, it follows that (4.3) provides an analytic continuation of $\Lambda_{\boldsymbol{r}}(s)$ into the half-plane $\left\{s \in \mathbb{C}:-\frac{1}{2}<\operatorname{Re}(s)\right\}$.

Next we observe that the function $u \rightarrow \varphi_{\boldsymbol{r}}(u) \mathrm{e}^{u / 2}$ belongs to $L^{2}\{[0, \infty)\}$. The Laguerre functions form a complete orthonormal system for $L^{2}\{[0, \infty)\}$. Therefore, we have

$$
\lim _{M \rightarrow \infty} \sum_{m=0}^{M} c_{m}(\boldsymbol{r}) \mathcal{L}_{m}(u)=\varphi_{\boldsymbol{r}}(u) \mathrm{e}^{u / 2}
$$


in $L^{2}$-norm on $[0, \infty)$, where the coefficients $c_{m}(\boldsymbol{r})$ are determined by

$$
\begin{aligned}
c_{m}(\boldsymbol{r}) & =\int_{0}^{\infty} \varphi_{\boldsymbol{r}}(u) \mathrm{e}^{u / 2} \mathcal{L}_{m}(u) \mathrm{d} u \\
& =\int_{0}^{\infty} \varphi_{\boldsymbol{r}}(u) L_{m}(u) \mathrm{d} u \\
& =\sum_{l=0}^{m}\left(\begin{array}{c}
m \\
l
\end{array}\right) \frac{(-1)^{l}}{l !}\left\{\int_{0}^{\infty} u^{l} \varphi_{\boldsymbol{r}}(u) \mathrm{d} u\right\} \\
& =\sum_{l=0}^{m}\left(\begin{array}{c}
m \\
l
\end{array}\right) \frac{(-1)^{l} \Lambda_{\boldsymbol{r}}(l)}{l !|\boldsymbol{r}|^{2 l}} .
\end{aligned}
$$

We note that $c_{0}(\boldsymbol{r})=1$, and

$$
c_{1}(\boldsymbol{r})=\int_{0}^{\infty} \varphi_{\boldsymbol{r}}(u)(1-u) \mathrm{d} u=\Lambda_{\boldsymbol{r}}(0)-|\boldsymbol{r}|^{-2} \Lambda_{\boldsymbol{r}}(1)=0 .
$$

Theorem 4.1. If $3 \leqslant N$, then

$$
\Lambda_{\boldsymbol{r}}(s)=|\boldsymbol{r}|^{2 s} \Gamma(s+1) \sum_{m=0}^{\infty} c_{m}(\boldsymbol{r})(-1)^{m}\left(\begin{array}{c}
s \\
m
\end{array}\right),
$$

where the sequence

$$
\Gamma(s+1) \sum_{m=0}^{M} c_{m}(\boldsymbol{r})(-1)^{m}\left(\begin{array}{c}
s \\
m
\end{array}\right), \quad M=0,1,2, \ldots,
$$

of analytic functions converges uniformly on each closed half-plane $\{s: \delta \leqslant \operatorname{Re}(s)\}$ with $-\frac{1}{2}<\delta$. Moreover, we have the identity

$$
\begin{aligned}
\Lambda_{\boldsymbol{r}}^{\prime}(0) & =2 \int_{(\mathbb{R} / \mathbb{Z})^{N}} \log \left|r_{1} e\left(x_{1}\right)+r_{2} e\left(x_{2}\right)+\cdots+r_{N} e\left(x_{N}\right)\right| \mathrm{d} \boldsymbol{x} \\
& =2 \log |\boldsymbol{r}|-\gamma-\sum_{m=2}^{\infty} \frac{c_{m}(\boldsymbol{r})}{m} .
\end{aligned}
$$

Proof. From (4.3) we get

$$
\begin{aligned}
\Lambda_{\boldsymbol{r}}(s)|\boldsymbol{r}|^{-2 s}= & \int_{0}^{\infty} u^{s}\left\{\varphi_{\boldsymbol{r}}(u) \mathrm{e}^{u / 2}\right\} \mathrm{e}^{-u / 2} \mathrm{~d} u \\
= & \int_{0}^{\infty} u^{s}\left\{\sum_{m=0}^{M} c_{m}(\boldsymbol{r}) \mathcal{L}_{m}(u)\right\} \mathrm{e}^{-u / 2} \mathrm{~d} u \\
& \quad+\int_{0}^{\infty} u^{s}\left\{\varphi_{\boldsymbol{r}}(u) \mathrm{e}^{u / 2}-\sum_{m=0}^{M} c_{m}(\boldsymbol{r}) \mathcal{L}_{m}(u)\right\} \mathrm{e}^{-u / 2} \mathrm{~d} u .
\end{aligned}
$$


If $-\frac{1}{2}<\delta \leqslant \operatorname{Re}(s)$, then, using Cauchy's inequality, we obtain the estimate

$$
\begin{aligned}
\mid \int_{0}^{\infty} u^{s}\left\{\varphi_{\boldsymbol{r}}(u) \mathrm{e}^{u / 2}\right. & \left.-\sum_{m=0}^{M} c_{m}(\boldsymbol{r}) \mathcal{L}_{m}(u)\right\}\left.\mathrm{e}^{-u / 2} \mathrm{~d} x\right|^{2} \\
& \leqslant\left\{\int_{0}^{\infty} u^{2 \sigma} \mathrm{e}^{-u} \mathrm{~d} u\right\}\left\{\int_{0}^{\infty}\left|\varphi_{\boldsymbol{r}}(v) \mathrm{e}^{v / 2}-\sum_{m=0}^{M} c_{m}(\boldsymbol{r}) \mathcal{L}_{m}(v)\right|^{2} \mathrm{~d} v\right\} \\
& \leqslant \Gamma(2 \delta+1) \sum_{m=M+1}^{\infty}\left|c_{m}(\boldsymbol{r})\right|^{2}
\end{aligned}
$$

Now, Lemma 2.1, (4.4) and the estimate (4.8) show that

$$
\begin{aligned}
\Lambda_{\boldsymbol{r}}(s)|\boldsymbol{r}|^{-2 s} & =\lim _{M \rightarrow \infty} \int_{0}^{\infty} u^{s}\left\{\sum_{m=0}^{M} c_{m}(\boldsymbol{r}) \mathcal{L}_{m}(u)\right\} \mathrm{e}^{-u / 2} \mathrm{~d} u \\
& =\lim _{M \rightarrow \infty} \sum_{m=0}^{M} c_{m}(\boldsymbol{r})\left\{\int_{0}^{\infty} u^{s} L_{m}(u) \mathrm{e}^{-u} \mathrm{~d} u\right\} \\
& =\Gamma(s+1) \lim _{M \rightarrow \infty} \sum_{m=0}^{M} c_{m}(\boldsymbol{r})(-1)^{m}\left(\begin{array}{c}
s \\
m
\end{array}\right)
\end{aligned}
$$

uniformly on $\{s: \delta \leqslant \operatorname{Re}(s)\}$. This verifies (4.6).

Because the series on the right-hand side of (4.6) converges uniformly in a neighbourhood of $s=0$, we can differentiate it term by term. This leads to the identity

$$
\begin{aligned}
\Lambda_{\boldsymbol{r}}^{\prime}(s)=|\boldsymbol{r}|^{2 s}(2 \log |\boldsymbol{r}|) \Gamma(s+1) & \sum_{m=0}^{\infty} c_{m}(\boldsymbol{r})(-1)^{m}\left(\begin{array}{c}
s \\
m
\end{array}\right) \\
& +|\boldsymbol{r}|^{2 s} \sum_{m=0}^{\infty} c_{m}(\boldsymbol{r}) \int_{0}^{\infty} u^{s}(\log u) L_{m}(u) \mathrm{e}^{-u} \mathrm{~d} u
\end{aligned}
$$

Finally, we set $s=0$ in (4.9) and appeal to Corollary 2.2. In this way we establish the identity (4.7).

Instead of proving (4.6) first, we can argue more directly to establish (4.7) as follows. From (4.3) we get

$$
\Lambda_{\boldsymbol{r}}^{\prime}(s)=|\boldsymbol{r}|^{2 s}(2 \log |\boldsymbol{r}|) \int_{0}^{\infty} u^{s} \varphi_{\boldsymbol{r}}(u) \mathrm{d} u+|\boldsymbol{r}|^{2 s} \int_{0}^{\infty} u^{s}(\log u) \varphi_{\boldsymbol{r}}(u) \mathrm{d} u
$$

and therefore

$$
\Lambda_{\boldsymbol{r}}^{\prime}(0)=2 \log |\boldsymbol{r}|+\int_{0}^{\infty}(\log u) \varphi_{\boldsymbol{r}}(u) \mathrm{d} u
$$

Now write

$$
g(u)=(\log u) \mathrm{e}^{-u / 2} \quad \text { and } \quad h_{\boldsymbol{r}}(u)=\varphi_{\boldsymbol{r}}(u) \mathrm{e}^{u / 2}
$$


so that the integral on the right-hand side of (4.10) can be viewed as an inner product

$$
\int_{0}^{\infty}(\log u) \varphi_{\boldsymbol{r}}(u) \mathrm{d} u=\left\langle g, h_{\boldsymbol{r}}\right\rangle
$$

in $L^{2}[0, \infty)$. We have already determined the coefficients in the expansion of both $g$ and $h_{\boldsymbol{r}}$ with respect to the complete orthonormal system of Laguerre functions. The coefficients $\left\langle g, \mathcal{L}_{m}\right\rangle$ were determined in $(2.3)$, and $\left\langle h_{\boldsymbol{r}}, \mathcal{L}_{m}\right\rangle=c_{m}(\boldsymbol{r})$. Hence we get

$$
\left\langle g, h_{\boldsymbol{r}}\right\rangle=\sum_{m=0}^{\infty}\left\langle g, \mathcal{L}_{m}\right\rangle\left\langle h_{\boldsymbol{r}}, \mathcal{L}_{m}\right\rangle=-\gamma-\sum_{m=2}^{\infty} \frac{c_{m}(\boldsymbol{r})}{m}
$$

as an application of Parseval's formula.

There is an alternative representation for the series on the right-hand side of (4.7) that can be derived from a generating function for the sequence $m \rightarrow c_{m}(\boldsymbol{r})$.

Theorem 4.2. If $3 \leqslant N$, then

$$
\begin{aligned}
\Lambda_{\boldsymbol{r}}^{\prime}(0) & =2 \int_{(\mathbb{R} / \mathbb{Z})^{N}} \log \left|r_{1} e\left(x_{1}\right)+r_{2} e\left(x_{2}\right)+\cdots+r_{N} e\left(x_{N}\right)\right| \mathrm{d} \boldsymbol{x} \\
& =2 \log |\boldsymbol{r}|-\gamma+2 \int_{0}^{\infty}\left\{\exp \left(-u^{2}\right)-\prod_{n=1}^{N} J_{0}\left(2 r_{n}|\boldsymbol{r}|^{-1} u\right)\right\} u^{-1} \mathrm{~d} u .
\end{aligned}
$$

Proof. Using (3.4) and the fact that $f_{\boldsymbol{r}}$ has compact support, we have

$$
\begin{aligned}
\prod_{n=1}^{N} J_{0}\left(2 r_{n}|\boldsymbol{t}|\right) & =\int_{\mathbb{R}^{2}} f_{\boldsymbol{r}}(\boldsymbol{x}) J_{0}(2|\boldsymbol{t}||\boldsymbol{x}|) \mathrm{d} \boldsymbol{x} \\
& =\sum_{m=0}^{\infty} \frac{(-1)^{m}}{(m !)^{2}}\left\{\int_{\mathbb{R}^{2}}|\boldsymbol{x}|^{2 m} f_{\boldsymbol{r}}(\boldsymbol{x}) \mathrm{d} \boldsymbol{x}\right\}|\boldsymbol{t}|^{2 m} \\
& =\sum_{m=0}^{\infty} \frac{(-1)^{m} \Lambda_{\boldsymbol{r}}(m)}{(m !)^{2}}|\boldsymbol{t}|^{2 m}
\end{aligned}
$$

Then we also get

$$
\begin{aligned}
\exp \left(|\boldsymbol{r}|^{2}|\boldsymbol{t}|^{2}\right) \prod_{n=1}^{N} J_{0}\left(2 r_{n}|\boldsymbol{t}|\right) & =\sum_{k=0}^{\infty} \sum_{l=0}^{\infty}\left\{\frac{|\boldsymbol{r}|^{2 k}|\boldsymbol{t}|^{2 k}}{k !}\right\}\left\{\frac{(-1)^{l} \Lambda_{\boldsymbol{r}}(l)|\boldsymbol{t}|^{2 l}}{(l !)^{2}}\right\} \\
& =\sum_{m=0}^{\infty}\left\{\sum_{l=0}^{m}\left(\begin{array}{c}
m \\
l
\end{array}\right) \frac{(-1)^{l} \Lambda_{\boldsymbol{r}}(l)}{l !|\boldsymbol{r}|^{2 l}}\right\} \frac{|\boldsymbol{r}|^{2 m}|\boldsymbol{t}|^{2 m}}{m !} \\
& =\sum_{m=0}^{\infty} \frac{c_{m}(\boldsymbol{r})|\boldsymbol{r}|^{2 m}|\boldsymbol{t}|^{2 m}}{m !},
\end{aligned}
$$

and therefore

$$
1-\exp \left(|\boldsymbol{t}|^{2}\right) \prod_{n=1}^{N} J_{0}\left(2 r_{n}|\boldsymbol{r}|^{-1}|\boldsymbol{t}|\right)=-\sum_{m=2}^{\infty} \frac{c_{m}(\boldsymbol{r})|\boldsymbol{t}|^{2 m}}{m !}
$$


We write $|\boldsymbol{t}|=u$ in (4.16) and conclude that

$$
\begin{aligned}
2 \int_{0}^{\infty}\left\{\exp \left(-u^{2}\right)-\prod_{n=1}^{N} J_{0}\left(2 r_{n}|\boldsymbol{r}|^{-1} u\right)\right\} u^{-1} \mathrm{~d} u & \\
& =-2 \int_{0}^{\infty}\left\{\sum_{m=2}^{\infty} \frac{c_{m}(\boldsymbol{r})}{m !} u^{2 m-1}\right\} \exp \left(-u^{2}\right) \mathrm{d} u
\end{aligned}
$$

Term-by-term integration on the right-hand side of (4.17) is justified because

$$
\left|\sum_{m=2}^{M} \frac{c_{m}(\boldsymbol{r})}{m !} u^{2 m-1}\right| \exp \left(-u^{2}\right) \leqslant \sum_{m=2}^{\infty} \frac{\left|c_{m}(\boldsymbol{r})\right|}{m !} u^{2 m-1} \exp \left(-u^{2}\right)
$$

and

$$
\begin{aligned}
\int_{0}^{\infty} \sum_{m=2}^{\infty} \frac{\left|c_{m}(\boldsymbol{r})\right|}{m !} u^{2 m-1} \exp \left(-u^{2}\right) \mathrm{d} u & =\sum_{m=2}^{\infty} \frac{\left|c_{m}(\boldsymbol{r})\right|}{m !} \int_{0}^{\infty} u^{2 m-1} \exp \left(-u^{2}\right) \mathrm{d} u \\
& \ll \sum_{m=2}^{\infty} \frac{\left|c_{m}(\boldsymbol{r})\right|}{m} \\
& \ll\left\{\sum_{m=2}^{\infty}\left|c_{m}(\boldsymbol{r})\right|^{2}\right\}^{1 / 2} \\
& <\infty
\end{aligned}
$$

Then the result of term-by-term integration on the right-hand side of (4.17) is

$$
-2 \int_{0}^{\infty}\left\{\sum_{m=2}^{\infty} \frac{c_{m}(\boldsymbol{r})}{m !} u^{2 m-1}\right\} \exp \left(-u^{2}\right) \mathrm{d} u=-\sum_{m=2}^{\infty} \frac{c_{m}(\boldsymbol{r})}{m} .
$$

The identity (4.13) follows now from (4.17) and (4.18).

The identity (4.15) provides an interesting representation for $c_{m}(\boldsymbol{r})$ in the special case $\boldsymbol{r}=\mathbf{1}_{N}$, where $\mathbf{1}_{N}$ is the vector in $\mathbb{R}^{N}$ having 1 in each coordinate. We find that

$$
\begin{aligned}
1+\sum_{m=2}^{\infty} \frac{c_{m}\left(\mathbf{1}_{N}\right) N^{m} z^{2 m}}{m !} & =\exp \left\{N z^{2}+N \log J_{0}(2 z)\right\} \\
& =\exp \left\{-N \sum_{l=2}^{\infty} l^{-1} \beta_{l} z^{2 l}\right\}
\end{aligned}
$$

If we expand the right-hand side of (4.19) and equate coefficients, we obtain the identity

$$
c_{m}\left(\mathbf{1}_{N}\right)=m ! \sum_{1 \leqslant L \leqslant[m / 2]}(-1)^{L} \frac{\tau(L, m)}{L !} N^{L-m} \text { for } m=2,3, \ldots,
$$


where

$$
\tau(L, m)=\sum_{\boldsymbol{k} \in T(L, m)} \frac{\beta_{k_{1}} \beta_{k_{2}} \cdots \beta_{k_{L}}}{k_{1} k_{2} \cdots k_{L}}
$$

and

$$
T(L, m)=\left\{\boldsymbol{k} \in \mathbb{Z}^{L}: 0 \leqslant k_{l} \text { and } k_{1}+k_{2}+\cdots+k_{L}=m\right\} .
$$

For example, we find that

$$
c_{2}\left(\mathbf{1}_{N}\right)=\frac{-1}{2 N}, \quad c_{3}\left(\mathbf{1}_{N}\right)=\frac{-2}{3 N^{2}}, \quad c_{4}\left(\mathbf{1}_{N}\right)=\frac{6 N-11}{8 N^{3}}, \quad c_{5}\left(\mathbf{1}_{N}\right)=\frac{50 N-57}{15 N^{4}} .
$$

This leads formally to

$$
-\sum_{m=2}^{\infty} \frac{c_{m}\left(\mathbf{1}_{N}\right)}{m}=\frac{1}{4 N}+\frac{5}{144 N^{2}}-\frac{1}{96 N^{3}}-\frac{1711}{86400 N^{4}}+\cdots,
$$

and we conjecture that (4.21) is in fact an asymptotic expansion (numerically, summing the series until the terms stop decreasing does seem to give the correct value up to roughly $N$ decimal places).

\section{An elementary inequality}

Lemma 5.1. Let $\boldsymbol{x}$ be a vector in $\mathbb{R}^{N}$ and let $y$ be a real number such that $0<y \leqslant$ $|\boldsymbol{x}|_{\infty}$. Assume that the coordinates of $\boldsymbol{x}$ are non-decreasing,

$$
0 \leqslant x_{1} \leqslant x_{2} \leqslant x_{3} \leqslant \cdots \leqslant x_{N}
$$

and set $x_{0}=0$. Let $M$ be the unique integer that satisfies $0 \leqslant M \leqslant N-1$ and $x_{M}<$ $y \leqslant x_{M+1}$. Then we have

$$
\int_{y^{-1}}^{\infty}\left(\prod_{n=1}^{N} \max \left\{1, x_{n} u\right\}\right)^{-1 / 2} u^{-1} \mathrm{~d} u \leqslant \frac{2}{N-M} \prod_{n=M+1}^{N}\left(\frac{y}{x_{n}}\right)^{1 / 2} .
$$

Proof. Set $R_{0}=0$ and

$$
R_{m}=\prod_{n=m}^{N}\left(\frac{x_{m}}{x_{n}}\right)^{1 / 2} \text { for } m=1,2,3, \ldots, N
$$

We find that

$$
\frac{R_{m+1}}{R_{m}}=\left(\frac{x_{m+1}}{x_{m}}\right)^{(N-m) / 2} \geqslant 1, \quad \text { for } m=1,2, \ldots, N-1,
$$

and therefore $0=R_{0}<R_{1} \leqslant R_{2} \leqslant \cdots \leqslant R_{N}=1$. Next we observe that

$$
\int_{x_{1}^{-1}}^{\infty}\left(\prod_{n=1}^{N} \max \left\{1, x_{n} u\right\}\right)^{-1 / 2} u^{-1} \mathrm{~d} u=\left\{\prod_{n=1}^{N} x_{n}\right\}^{-1 / 2} \int_{x_{1}^{-1}}^{\infty} u^{-1-N / 2} \mathrm{~d} u=\frac{2}{N}\left\{R_{1}-R_{0}\right\},
$$


and for each $m=1,2, \ldots, N-1$ we have

$$
\begin{aligned}
\int_{x_{m+1}^{-1}}^{x_{m}^{-1}}\left(\prod_{n=1}^{N} \max \left\{1, x_{n} u\right\}\right)^{-1 / 2} u^{-1} \mathrm{~d} u & \\
& =\left\{\prod_{n=m+1}^{N} x_{n}\right\}^{-1 / 2} \int_{x_{m+1}^{-1}}^{x_{m}^{-1}} u^{-1-(N-m) / 2} \mathrm{~d} u \\
& =\frac{2}{N-m}\left\{\prod_{n=m+1}^{N} x_{n}\right\}^{-1 / 2}\left\{\left(x_{m+1}\right)^{(N-m) / 2}-\left(x_{m}\right)^{(N-m) / 2}\right\} \\
& =\frac{2}{N-m}\left\{\prod_{n=m+1}^{N}\left(\frac{x_{m+1}}{x_{n}}\right)^{1 / 2}-\prod_{n=m+1}^{N}\left(\frac{x_{m}}{x_{n}}\right)^{1 / 2}\right\} \\
& =\frac{2}{N-m}\left\{R_{m+1}-R_{m}\right\}
\end{aligned}
$$

In a similar manner, if $0<y \leqslant x_{1}$, we find that

$$
\int_{y^{-1}}^{\infty}\left(\prod_{n=1}^{N} \max \left\{1, x_{n} u\right\}\right)^{-1 / 2} u^{-1} \mathrm{~d} u=\frac{2}{N}\left\{\prod_{n=1}^{N}\left(\frac{y}{x_{n}}\right)^{1 / 2}-R_{0}\right\},
$$

and if $x_{m}<y \leqslant x_{m+1}$, where $m=1,2, \ldots, N-1$, then

$$
\int_{y^{-1}}^{x_{m}^{-1}}\left(\prod_{n=1}^{N} \max \left\{1, x_{n} u\right\}\right)^{-1 / 2} u^{-1} \mathrm{~d} u=\frac{2}{N-m}\left\{\prod_{n=m+1}^{N}\left(\frac{y}{x_{n}}\right)^{1 / 2}-R_{m}\right\} .
$$

Now let $M$ be as in the statement of the lemma. Then we have

$$
\begin{aligned}
\int_{y^{-1}}^{\infty}\left(\prod_{n=1}^{N} \max \left\{1, x_{n} u\right\}\right)^{-1 / 2} u^{-1} \mathrm{~d} u & \int_{y^{-1}}^{x_{M}^{-1}}\left(\prod_{n=1}^{N} \max \left\{1, x_{n} u\right\}\right)^{-1 / 2} u^{-1} \mathrm{~d} u \\
& +\sum_{m=0}^{M-1} \int_{x_{m+1}^{-1}}^{x_{m}^{-1}}\left(\prod_{n=1}^{N} \max \left\{1, x_{n} u\right\}\right)^{-1 / 2} u^{-1} \mathrm{~d} u \\
& =\frac{2}{N-M}\left\{\prod_{n=M+1}^{N}\left(\frac{y}{x_{n}}\right)^{1 / 2}-R_{M}\right\}+\sum_{m=0}^{M-1} \frac{2}{N-m}\left\{R_{m+1}-R_{m}\right\} \\
& =\frac{2}{N-M} \prod_{n=M+1}^{N}\left(\frac{y}{x_{n}}\right)^{1 / 2}+2 \sum_{m=0}^{M-1} \frac{R_{m+1}}{N-m}-2 \sum_{m=0}^{M} \frac{R_{m}}{N-m} \\
& =\frac{2}{N-M} \prod_{n=M+1}^{N}\left(\frac{y}{x_{n}}\right)^{1 / 2}-2 \sum_{m=1}^{M} \frac{R_{m}}{(N-m+1)(N-m)} \\
& \leqslant \frac{2}{N-M} \prod_{n=M+1}^{N}\left(\frac{y}{x_{n}}\right)^{1 / 2} .
\end{aligned}
$$

This proves the lemma. 
We note that there is equality in the inequality (5.6) whenever $0<y \leqslant x_{1}$. In particular, there is equality in (5.6) in the case where $x_{1}=x_{2}=\cdots=x_{N}$.

\section{Proof of Theorem 1.2}

The value of the Mahler measure $m\left(L_{\boldsymbol{a}}\right)$ is unchanged if we replace the coefficients $a_{n}$ with $\left|a_{n}\right|=r_{n}$ for each $n=1,2, \ldots, N$. Then by Theorem 4.2 it suffices to bound the integral

$$
\int_{0}^{\infty}\left\{\exp \left(-u^{2}\right)-\prod_{n=1}^{N} J_{0}\left(2 r_{n}|\boldsymbol{r}|^{-1} u\right)\right\} u^{-1} \mathrm{~d} u,
$$

which appears on the right-hand side of (4.13). As the value of this integral is a homogeneous function of $\boldsymbol{r}$ with degree zero, we may assume without loss of generality that $|\boldsymbol{r}|=1$. We also write $R=|\boldsymbol{r}|_{\infty}^{-1}$ so that $1 \leqslant R \leqslant N^{1 / 2}$. We then have

$$
\int_{0}^{\infty}\left\{\exp \left(-u^{2}\right)-\prod_{n=1}^{N} J_{0}\left(2 r_{n} u\right)\right\} u^{-1} \mathrm{~d} u=I_{1}+I_{2}+I_{3},
$$

where

$$
\begin{aligned}
& I_{1}=\int_{0}^{R}\left\{\exp \left(-u^{2}\right)-\prod_{n=1}^{N} J_{0}\left(2 r_{n} u\right)\right\} u^{-1} \mathrm{~d} u \\
& I_{2}=\int_{R}^{\infty} \exp \left(-u^{2}\right) u^{-1} \mathrm{~d} u
\end{aligned}
$$

and

$$
I_{3}=-\int_{R}^{\infty}\left\{\prod_{n=1}^{N} J_{0}\left(2 r_{n} u\right)\right\} u^{-1} \mathrm{~d} u .
$$

In order to estimate $I_{1}$ we appeal to (2.5). We conclude that

$$
\begin{aligned}
0 \leqslant I_{1} & \leqslant \frac{1}{2} \int_{0}^{R} \exp \left\{-u^{2}\right\} S_{2}(u \boldsymbol{r}) u^{-1} \mathrm{~d} u \\
& \leqslant \frac{1}{2}\left(\int_{0}^{\infty} u^{3} \exp \left\{-u^{2}\right\} \mathrm{d} u\right) S_{2}(\boldsymbol{r}) \\
& =\frac{1}{4} \sum_{n=1}^{N} r_{n}^{4} .
\end{aligned}
$$

The integral $I_{2}$ is trivial to estimate:

$$
0 \leqslant I_{2} \leqslant R^{-2} \int_{R}^{\infty} u \mathrm{e}^{-u^{2}} \mathrm{~d} u=\frac{1}{2} R^{-2} \mathrm{e}^{-R^{2}}
$$


In order to estimate $I_{3}$ we use the inequality (3.5). Then we get

$$
\begin{aligned}
\left|I_{3}\right| & \leqslant \int_{R}^{\infty}\left\{\prod_{n=1}^{N}\left|J_{0}\left(2 r_{n} u\right)\right|\right\} u^{-1} \mathrm{~d} u \\
& \leqslant \int_{R}^{\infty}\left(\prod_{n=1}^{N} \max \left\{1, \pi\left|r_{n}\right| u\right\}\right)^{-1 / 2} u^{-1} \mathrm{~d} u \\
& =\int_{\pi R}^{\infty}\left(\prod_{n=1}^{N} \max \left\{1,\left|r_{n}\right| u\right\}\right)^{-1 / 2} u^{-1} \mathrm{~d} u .
\end{aligned}
$$

We may assume without loss of generality that $0<r_{1} \leqslant r_{2} \leqslant \cdots \leqslant r_{N}$. Then we apply the inequality (5.1) with $M$ the unique integer satisfying $0 \leqslant M \leqslant N-1$ and

$$
\left|r_{M}\right|<(\pi R)^{-1}=\pi^{-1}|\boldsymbol{r}|_{\infty} \leqslant\left|r_{M+1}\right| .
$$

Alternatively, we apply (5.1) with

$$
N-M=\mid\left\{n: 1 \leqslant n \leqslant N \text { and }|\boldsymbol{r}|_{\infty} \leqslant \pi\left|r_{n}\right|\right\} \mid .
$$

We note that the cardinality of the set on the right-hand side of (6.5) is at least 1 . Thus the estimates (5.1) and (6.4) imply that

$$
\left|I_{3}\right| \leqslant 2\left|\left\{n: 1 \leqslant n \leqslant N,|\boldsymbol{r}|_{\infty} \leqslant \pi\left|r_{n}\right|\right\}\right|^{-1} .
$$

If we combine (6.2), (6.3) and (6.6), we find that

$$
\begin{aligned}
-2\left|\left\{n: 1 \leqslant n \leqslant N,|\boldsymbol{r}|_{\infty} \leqslant \pi\left|r_{n}\right|\right\}\right|^{-1} \leqslant & \int_{0}^{\infty}\left\{\exp \left(-u^{2}\right)-\prod_{n=1}^{N} J_{0}\left(2 r_{n}|\boldsymbol{r}|^{-1} u\right)\right\} u^{-1} \mathrm{~d} u \\
\leqslant & \frac{1}{4}\left\{\sum_{n=1}^{N} r_{n}^{4}\right\}+\frac{1}{2}|\boldsymbol{r}|_{\infty}^{2} \exp \left\{-|\boldsymbol{r}|_{\infty}^{-2}\right\} \\
& +2\left|\left\{n: 1 \leqslant n \leqslant N,|\boldsymbol{r}|_{\infty} \leqslant \pi\left|r_{n}\right|\right\}\right|^{-1} .
\end{aligned}
$$

The expression on the right-hand side of (6.7) can be simplified. Observe that

$$
\left|\left\{n: 1 \leqslant n \leqslant N,|\boldsymbol{r}|_{\infty} \leqslant \pi\left|r_{n}\right|\right\}\right| \leqslant \sum_{n=1}^{N}\left(\frac{\pi\left|r_{n}\right|}{|\boldsymbol{r}|_{\infty}}\right)^{2}=\pi^{2}|\boldsymbol{r}|_{\infty}^{-2}
$$

and therefore

$$
|\boldsymbol{r}|_{\infty}^{2} \leqslant \pi^{2}\left|\left\{n: 1 \leqslant n \leqslant N,|\boldsymbol{r}|_{\infty} \leqslant \pi\left|r_{n}\right|\right\}\right|^{-1} .
$$

Thus (6.7) implies that

$$
\begin{aligned}
& \int_{0}^{\infty}\left\{\exp \left(-u^{2}\right)-\prod_{n=1}^{N} J_{0}\left(2 r_{n}|\boldsymbol{r}|^{-1} u\right)\right\} u^{-1} \mathrm{~d} u \\
& \quad \leqslant \frac{1}{4}\left\{\sum_{n=1}^{N} r_{n}^{4}\right\}+4\left|\left\{n: 1 \leqslant n \leqslant N,|\boldsymbol{r}|_{\infty} \leqslant \pi\left|r_{n}\right|\right\}\right|^{-1}
\end{aligned}
$$

The inequality (1.8) follows now from (4.13), (6.7) and (6.8). 


\section{Proof of Theorem 1.3}

We apply (4.1) with $T(x)=\log ^{+} x$ and find that

$$
\begin{aligned}
\int_{(\mathbb{R} / \mathbb{Z})^{N}} \log ^{+}\left|a_{1} e\left(\theta_{1}\right)+a_{2} e\left(\theta_{2}\right)+\cdots+a_{N} e\left(\theta_{N}\right)\right| \mathrm{d} \boldsymbol{\theta} & =\int_{\mathbb{R}^{2}} f_{\boldsymbol{r}}(\boldsymbol{x}) \log ^{+}|\boldsymbol{x}| \mathrm{d} \boldsymbol{x} \\
& =2 \pi \int_{0}^{\infty} u f_{\boldsymbol{r}}(u) \log ^{+} u \mathrm{~d} u \\
& =\int_{1}^{|\boldsymbol{r}|_{1}}\left(1-\tilde{F}_{\boldsymbol{r}}(u)\right) u^{-1} \mathrm{~d} u .
\end{aligned}
$$

As before let $0<\alpha_{1}<\alpha_{2}<\cdots$ denote the consecutive positive zeros of the Bessel function $J_{0}(z)$. We recall the basic identity $[\mathbf{1 3}$, Chapter $18, \S 1]$

$$
2 \int_{0}^{1} u J_{0}\left(\alpha_{l} u\right) J_{0}\left(\alpha_{m} u\right) \mathrm{d} u= \begin{cases}0 & \text { if } l \neq m, \\ J_{1}\left(\alpha_{l}\right)^{2} & \text { if } l=m .\end{cases}
$$

Write $\left|a_{n}\right|=r_{n}$ and $R=|\boldsymbol{a}|_{1}=|\boldsymbol{r}|_{1}$. Then define functions $\Psi_{l}:[0, R] \rightarrow \mathbb{R}$ for $l=1,2, \ldots$ by

$$
\Psi_{l}(u)=R^{-1} J_{1}\left(\alpha_{l}\right)^{-1}(2 u)^{1 / 2} J_{0}\left(\alpha_{l} R^{-1} u\right) .
$$

From (7.2) we conclude that the functions $\left\{\Psi_{1}(u), \Psi_{2}(u), \ldots\right\}$ form an orthonormal system in $L^{2}\{[0, R]\}$, and this system is complete [13, Chapter 18, $\left.\S 24\right]$. Define functions $G_{\boldsymbol{r}}:[0, R] \rightarrow \mathbb{R}$ and $H_{\boldsymbol{r}}:[0, R] \rightarrow \mathbb{R}$ by

$$
G_{\boldsymbol{r}}(u)= \begin{cases}0 & \text { if } 0 \leqslant u<1 \\ u^{-3 / 2} & \text { if } 1 \leqslant u \leqslant R\end{cases}
$$

and

$$
H_{\boldsymbol{r}}(u)=\left(1-\tilde{F}_{\boldsymbol{r}}(u)\right) u^{1 / 2}
$$

It follows that

$$
\left\langle G_{\boldsymbol{r}}, \Psi_{l}\right\rangle=2^{1 / 2} R^{-1} J_{1}\left(\alpha_{l}\right)^{-1} \int_{1}^{R} J_{0}\left(\alpha_{l} R^{-1} u\right) \mathrm{d} u,
$$

and using Lemma 3.3 we find that

$$
\left\langle H_{\boldsymbol{r}}, \Psi_{l}\right\rangle=2^{1 / 2} \alpha_{l}^{-1} J_{1}\left(\alpha_{l}\right)^{-1} \sum_{m=1}^{N} r_{m} J_{1}\left(\alpha_{l} q_{m}\right) \prod_{\substack{n \neq 1 \\ n \neq m}}^{N} J_{0}\left(\alpha_{l} q_{n}\right) .
$$

It now follows using (7.3), (7.4) and Parseval's identity that

$$
\begin{aligned}
\int_{1}^{|\boldsymbol{r}|_{1}}\left(1-\tilde{F}_{\boldsymbol{r}}(u)\right) u^{-1} \mathrm{~d} u & =\left\langle G_{\boldsymbol{r}}, H_{\boldsymbol{r}}\right\rangle \\
& =\sum_{l=1}^{\infty}\left\langle G_{\boldsymbol{r}}, \Psi_{l}\right\rangle\left\langle H_{\boldsymbol{r}}, \Psi_{l}\right\rangle \\
& =\sum_{l=1}^{\infty} \psi(\boldsymbol{a}, l) \int_{1}^{|\boldsymbol{a}|_{1}} J_{0}\left(\alpha_{l}|\boldsymbol{a}|_{1}^{-1} u\right) u^{-1} \mathrm{~d} u .
\end{aligned}
$$

We get (1.14) in the statement of Theorem 1.3 by combining (7.1) and (7.5). 
In the special case $r_{1}=r_{2}=\cdots=r_{N}$, the Fourier-Bessel expansion arising from (7.4) was first obtained by Bennett [2]. The general case considered here is also recorded in [2]. However, [2, Eqn (12)] contains a non-trivial typographical error.

\section{Proof of Corollary 1.4}

For $0<u$ we define

$$
A_{0}(u)=\left(\frac{2}{\pi u}\right)^{1 / 2} \cos (u-\pi / 4)
$$

Then the estimate

$$
J_{0}(u)=A_{0}(u)+O\left(u^{-3 / 2}\right) \quad \text { as } u \rightarrow \infty
$$

is well known (see [11, Lemma 3.11] or [13, Chapter VII]). In fact, it can be shown that

$$
\left|J_{0}(u)-A_{0}(u)\right| \leqslant \frac{2}{7} u^{-3 / 2} \text { for } \frac{1}{2} \leqslant u,
$$

and this can be used to verify the inequality

$$
\int_{0}^{\infty} u\left\{J_{0}(u)-A_{0}(u)\right\}^{2} \mathrm{~d} u \leqslant 1 .
$$

We also find that

$$
\alpha_{l} \leqslant \alpha_{l}+\sin ^{2} \alpha_{l}=\pi \int_{0}^{\alpha_{l}} u A_{0}(u)^{2} \mathrm{~d} u .
$$

Then using (7.2), (8.2) and Minkowski's inequality we have

$$
\begin{aligned}
\left(\frac{\alpha_{l}}{\pi}\right)^{1 / 2} & \leqslant\left\{\int_{0}^{\alpha_{l}} u J_{0}(u)^{2} \mathrm{~d} u\right\}^{1 / 2}+\left\{\int_{0}^{\alpha_{l}} u\left\{J_{0}(u)-A_{0}(u)\right\}^{2} \mathrm{~d} u\right\}^{1 / 2} \\
& \leqslant\left\{\alpha_{l}^{2} \int_{0}^{1} u J_{0}\left(\alpha_{l} u\right)^{2} \mathrm{~d} u\right\}^{1 / 2}+1 \\
& =2^{-1 / 2} \alpha_{l}\left|J_{1}\left(\alpha_{l}\right)\right|+1
\end{aligned}
$$

and therefore

$$
\pi^{-1 / 2}-\alpha_{l}^{-1 / 2} \leqslant\left(\frac{1}{2} \alpha_{l}\right)^{1 / 2}\left|J_{1}\left(\alpha_{l}\right)\right| .
$$

If $16 \leqslant \alpha_{l}$, that is, if $6 \leqslant l$, then (8.4) implies that

$$
\alpha_{l}^{-1} J_{1}\left(\alpha_{l}\right)^{-2} \leqslant 8
$$

Next we use the inequality (3.5) and the identity

$$
J_{0}(x)^{2}+J_{1}(x)^{2}=2 \int_{0}^{1} u J_{0}(x u)^{2} \mathrm{~d} u
$$

to show that

$$
J_{1}(x)^{2} \leqslant\left(\frac{4}{\pi|x|}\right) \text { for all } x \in \mathbb{R}, x \neq 0 .
$$


Then we find that

$$
\left|\sum_{m=1}^{N} q_{m} J_{1}\left(\alpha_{l} q_{m}\right) \prod_{\substack{n \neq 1 \\ n \neq m}}^{N} J_{0}\left(\alpha_{l} q_{n}\right)\right| \leqslant 2^{1 / 2} \prod_{n=1}^{N}\left(\frac{2}{\pi q_{n} \alpha_{l}}\right)^{1 / 2}=2^{1 / 2}\left(\frac{2}{\pi Q \alpha_{l}}\right)^{N / 2} .
$$

We also use the inequality (8.1) to prove that

$$
\left|\int_{1}^{|\boldsymbol{a}|_{1}} J_{0}\left(\alpha_{l}|\boldsymbol{a}|_{1}^{-1} u\right) u^{-1} \mathrm{~d} u\right| \leqslant 3|\boldsymbol{a}|_{1}^{3 / 2} \alpha_{l}^{-3 / 2}
$$

By combining (8.5), (8.6) and (8.7) we arrive at the estimate

$$
\left|\psi(\boldsymbol{a}, l) \int_{1}^{|\boldsymbol{a}|_{1}} J_{0}\left(\alpha_{l}|\boldsymbol{a}|_{1}^{-1} u\right) u^{-1} \mathrm{~d} u\right| \leqslant 70|\boldsymbol{a}|_{1}^{3 / 2}\left(\frac{2}{\pi Q}\right)^{N / 2} \alpha_{l}^{-(N+3) / 2},
$$

provided $6 \leqslant l$.

Finally, it is known $[\mathbf{1 3}$, Chapter $15, \S 32]$ that the positive zeros of the Bessel function $J_{0}(z)$ satisfy the inequality

$$
\pi\left(l-\frac{1}{4}\right) \leqslant \alpha_{l} \leqslant \pi\left(l-\frac{1}{8}\right) \quad \text { for } l=1,2, \ldots
$$

It follows that

$$
\begin{aligned}
\sum_{l=L+1}^{\infty} \alpha_{l}^{-(N+3) / 2} & \leqslant \pi^{-(N+3) / 2} \sum_{l=L+1}^{\infty}\left(l-\frac{1}{4}\right)^{-(N+3) / 2} \\
& \leqslant \pi^{-(N+3) / 2} \int_{L+(1 / 2)}^{\infty}\left(t-\frac{1}{4}\right)^{-(N+3) / 2} \mathrm{~d} t \\
& \leqslant \pi^{-(N+3) / 2}\left(\frac{2}{N+1}\right) L^{-(N+1) / 2} .
\end{aligned}
$$

The bound (1.15) in the statement of Corollary 1.4 follows by combining (8.8) and (8.9).

Acknowledgements. The research of F.R.-V. was supported in part by the National Science Foundation (DMS-99-70109). The research of R.T. was supported in part by Conicet and Proyecto Fomec-UNL. The research of J.D.V. was supported in part by the National Science Foundation (DMS-00-88915).

\section{References}

1. A. Beilinson, Applications of algebraic $K$-theory to algebraic geometry and number theory, Parts I and II, in Contemporary mathematics, vol. 55, pp. 1-34 (Providence, RI, American Mathematical Society, 1986).

2. W. R. Bennett, Distribution of the sum of randomly phased components, Q. J. Mech. Appl. Math. 5 (1947), 385-393.

3. S. BlOCh AND D. GRAYSON, $K_{2}$ and $L$-functions of elliptic curves: computer calculations, in Contemporary mathematics, vol. 55, pp. 79-88 (Providence, RI, American Mathematical Society, 1986). 
4. D. Boyd, Speculations concerning the range of Mahler's measure, Can. Math. Bull. 24 (1981), 453-469.

5. D. Boyd, Mahler's measure and special values of $L$-functions, Exp. Math. 7 (1988), 37-82.

6. C. Deninger, Deligne periods of mixed motives, $K$-theory and the entropy of certain $\mathbb{Z}^{n}$-actions, J. Am. Math. Soc. 10 (1997), 259-281.

7. G. Everest AND T. WARD, Heights of polynomials and entropy in algebraic dynamics (Springer, 1999).

8. G. Myerson And C. J. Smyth, On measures of polynomials in several variables; corrigendum, Bull. Austral. Math. Soc. 26 (1982), 317-319.

9. F. Rodriguez-Villegas, Modular Mahler measures, I, in Topics in number theory (ed. S. D. Ahlgren, G. E. Andrews and K. Ono), pp. 17-48 (Deventer, Kluwer, 1999).

10. C. J. Sмyтн, On measures of polynomials in several variables, Bull. Austral. Math. Soc. 23 (1981), 49-63.

11. E. M. Stein AND G. Weiss, Introduction to Fourier analysis on euclidean spaces (Princeton University Press, 1971).

12. G. Szegö, Orthogonal polynomials, Colloquium Publications, vol. 23, 4th edn (Providence, RI, American Mathematical Society, 1978).

13. N. G. WAtson, A treatise on the theory of bessel functions, 2nd edn (Cambridge University Press, 1944). 